La planificación estratégica de los procesos de evaluación institucional y acreditación de las carreras de grado de la UNLP como política permanente de mejoramiento

Magalí Catino, Marina Semper

Revista ES (en y sobre Educación Superior)

Vol.1, N¹-2 / Fecha de publicación: 27/12/2021

e-ISSN: 2718-6539

https://revistas.unlp.edu.ar/ES/index

IIES - Facultad de Odontología

DOI: https://doi.org/10.24215/27186539e008

\title{
La planificación estratégica de los procesos de evaluación institucional y acreditación de las carreras de grado de la UNLP como política permanente de mejoramiento
}

The strategic planning of the institutional evaluation and accreditation processes of the undergraduate courses of the UNLP as a permanent improvement policy

O planejamento estratégico dos processos de avaliação institucional e credenciamento dos cursos de graduação da UNLP como política de melhoria permanente

Profesora Magalí Catino Directora de Evaluación Institucional - UNLP https://orcid.org/0000-0002-9402-4809 magali.catino@gmail.com 


\section{Arquitecta Marina Semper}

Dirección de Evaluación Institucional - UNLP marina.semper@presi.unlp.edu.ar

\section{Resumen}

La evaluación Institucional y la acreditación de carreras de grado son instancias fundamentales para reflexionar sobre los procesos internos de la vida universitaria, atendiendo su complejidad y coyuntura, pero con la mirada puesta en el continuo mejoramiento de las prácticas institucionales. El presente trabajo ordena las características centrales desde una mirada integrada de ambos procesos y a partir de allí se propone el desarrollo de un proyecto de innovación para la planificación estratégica de los procesos de evaluación institucional y acreditación de carreras de grado, como política permanente de mejoramiento en el marco del Plan Estratégico de la Universidad Nacional de La Plata. Se abordan esquemáticamente: la propuesta de un diseño metodológico de producción de información permanente de las distintas dimensiones para los procesos de evaluación institucional y de acreditación de las carreras de grado, las estrategias que permitan desarrollar los lineamientos y diseños de políticas sustantivas de la autoevaluación institucional para el mejoramiento, y los materiales de capacitación para los procesos de autoevaluación institucional continua y de acreditación de carreras de grado.

\section{Abstract}


Institutional evaluation and the accreditation of undergraduate courses are fundamental instances to reflect on the internal processes of university life, taking into account its complexity and situation, but with an eye on the continuous improvement of institutional practices. The present work orders the central characteristics from an integrated view of both processes and from there the development of an innovation project for the strategic planning of the processes of institutional evaluation and accreditation of undergraduate courses is proposed, as a permanent policy of improvement in the framework of the Strategic Plan of the National University of La Plata. They are approached schematically: the proposal of a methodological design for the production of permanent information of the different dimensions for the processes of institutional evaluation and accreditation of undergraduate courses, the strategies that allow the development of guidelines and substantive policy designs for institutional self-evaluation. for improvement, and training materials for continuous institutional self-evaluation and degree course accreditation processes.

\section{Resumo}

A avaliação institucional e o credenciamento dos cursos de graduação são instâncias fundamentais para refletir sobre os processos internos da vida universitária, considerando sua complexidade e situação, mas com vistas à melhoria contínua das práticas institucionais. O presente trabalho ordena as características centrais numa perspectiva integrada de ambos os processos e a partir daí se propõe o desenvolvimento de um projeto de inovação para o planejamento estratégico dos processos de avaliação institucional e credenciamento de cursos de graduação, como uma política permanente de 
aprimoramento do quadro. do Plano Estratégico da Universidade Nacional de La Plata. São abordados esquematicamente: a proposta de um desenho metodológico para a produção de informações permanentes sobre as diferentes dimensões dos processos de avaliação e acreditação institucional dos cursos de graduação, as estratégias que permitem o desenvolvimento de diretrizes e desenhos de políticas substantivas para a autoavaliação institucional. para melhoria e materiais de treinamento para processos contínuos de autoavaliação institucional e credenciamento de cursos de graduação.

\section{Palabras clave}

Evaluación institucional, acreditación, carreras de grado.

\section{Keywords}

Institutional evaluation, accreditation, degree courses.

\section{Palavras chave}

Avaliação institucional, credenciamento, cursos de graduação.

La evaluación Institucional y la acreditación de carreras de grado son instancias fundamentales para reflexionar sobre los procesos internos de la vida universitaria, atendiendo su complejidad y coyuntura, pero con la mirada puesta en el continuo mejoramiento de las prácticas institucionales.

En el año 2009 la Universidad concretó, a través del Informe Final de la CONEAU, la primera Evaluación Institucional. El mismo fue producto de un largo proceso que llevó casi diez años (habiendo comenzado en 2001) 
En el año 2001, se concreta en la UNLP el primer proceso de acreditación de carreras de grado con Medicina. A la fecha, la universidad lleva implementados 74 procesos de acreditación de carreras de grado (algunas de cuyas carreras lo han sido varias veces) que implican a 12 de las 17 Facultades.

Por otro lado, la Universidad comenzó en el año 2018, su segundo proceso de Evaluación Institucional, que sumado a esta trayectoria de acreditaciones de carreras de grado permite visibilizar que la cultura institucional de la Evaluación para el mejoramiento, ya es parte de la gestión permanente de la Universidad, ya sea a través de las acreditaciones de carreras de grado como por la Evaluación Institucional del conjunto de la UNLP.

\section{Sobre el proceso de evaluación institucional y acreditación de las carreras de grado}

La Ley $N^{\circ} 24.521$ de Educación Superior (LES) sancionada el 20 de julio de 1995, en los artículos 44ํy y 43ํ de la sección 3 correspondiente a evaluación y acreditación y la sección 2 respecto del régimen de títulos, establece la regulación específica sobre los procesos de Evaluación Institucional y de acreditación de carreras de grado.

Respecto de la Evaluación Institucional la LES lo define en su Artículo $44^{\circ}$ "Las instituciones universitarias deberán asegurar el funcionamiento de instancias internas de evaluación institucional, que tendrán por objeto analizar los logros y dificultades en el cumplimiento de sus funciones, así como sugerir medidas para su mejoramiento. Las autoevaluaciones se complementarán con evaluaciones externas, que se harán como mínimo cada seis (6) años, en el marco de los objetivos definidos por cada institución. Abarcará las funciones de docencia, investigación y extensión, y en el caso de las instituciones 
universitarias nacionales, también la gestión institucional. Las evaluaciones externas estarán a cargo de la Comisión Nacional de Evaluación y Acreditación Universitaria o de entidades privadas constituidas con ese fin, conforme se prevé en el artículo 45, en ambos casos con la participación de pares académicos de reconocida competencia. Las recomendaciones para el mejoramiento institucional que surjan de las evaluaciones tendrán carácter público".

En relación a la acreditación de carreras de grado la LES define en su Artículo 43: "Cuando se trate de títulos correspondientes a profesiones reguladas por el Estado, cuyo ejercicio pudiera comprometer el interés público poniendo en riesgo de modo directo la salud, la seguridad, los derechos, los bienes o la formación de los habitantes, se requerirá que se respeten, además de la carga horaria a la que hace referencia el artículo anterior $\left(42^{\circ}\right)$, los siguientes requisitos: a) Los planes de estudio deberán tener en cuenta los contenidos curriculares básicos y los criterios sobre la intensidad de la formación práctica que establezca el Ministerio de Cultura y Educación, en acuerdo con el Consejo de Universidades; b) Las carreras respectivas deberán ser acreditadas periódicamente por la Comisión Nacional de Evaluación y Acreditación Universitaria o por entidades privadas constituidas con ese fin debidamente reconocidas. El Ministerio de Cultura y Educación determinará con criterio restrictivo, en acuerdo con el Consejo de Universidades, la nómina de tales títulos, así como las actividades profesionales reservadas exclusivamente para ellos".

La LES también crea la CONEAU, en su Artículo 46" :La Comisión Nacional de Evaluación y Acreditación Universitaria es un organismo descentralizado, que funciona en jurisdicción del Ministerio de Cultura y Educación”. 
En función de esa disposición, en agosto de 1996 se concreta la creación de la Comisión Nacional de Evaluación y Acreditación Universitaria (CONEAU), que es el organismo encargado de los procesos de evaluación institucional y acreditación de las carreras de grado incorporadas al artículo $43^{\circ}$ y de posgrado.

\section{Evaluación Institucional}

En nuestro país, el sistema de evaluación es obligatorio y cada institución universitaria, así como cada carrera de grado incluida en el artículo $43^{\circ} \mathrm{o}$ de posgrado, y a partir de 2018, el Sistema de Educación a Distancia (SIED), debe llevar adelante un proceso periódico de evaluación o acreditación. Cada institución universitaria debe responder a un proceso de evaluación institucional, según dispone el artículo 44ํ de la misma norma. Dicho proceso se lleva a cabo periódicamente (cada 6 años) y tiene como parámetro el propio desarrollo institucional definido por su Ley de creación y su Estatuto vigente, así como su política y planificación estratégica. Para los procesos de acreditación la base o parámetro es en relación con el cumplimiento de estándares, los cuales son definidos por el Ministerio de Educación, en acuerdo con el Consejo de Universidades (CU). Este último está integrado por representantes del Consejo Interuniversitario Nacional (CIN), el Consejo de Rectores de Universidades Privadas (CRUP) y los Consejos Regionales de Planificación de la Educación Superior (CPRES).

De acuerdo con lo dispuesto en la Ley de Educación Superior 24.521 en su artículo 44ํㅜ, desde el año 1997 se comenzó con la implementación de las Evaluaciones Institucionales en Argentina. Dicho proceso involucra un primer momento de autoevaluación y un segundo momento de evaluación externa de 
pares, organizado por la Comisión Nacional de Evaluación y Acreditación Universitaria (CONEAU), también creada por la LES, como fue referido anteriormente.

El desarrollo de esta experiencia ha sido paulatino y ordenado de acuerdo a procedimientos definidos por la CONEAU, la cual ha generado normativas que se fueron modificando en el tiempo y que regulan el mecanismo de manera integral. Las pautas que orientan el trabajo involucran criterios e instrumentos que regulan tanto los procesos de autoevaluación institucional, como los de evaluación externa.

En 2011 la CONEAU aprueba la Resolución № 382 "Criterios y procedimientos para la evaluación externa", que detalla la información básica que debe contemplar la autoevaluación institucional. Este documento normativo establece la relación entre la evaluación institucional y otras evaluaciones, como la acreditación de carreras de grado y de posgrado, también llevadas a cabo por la CONEAU y establece criterios generales para la evaluación externa, por parte de pares.

Posteriormente, en el año 2013, a partir de la firma de un Acta de Colaboración entre la Secretaría de Políticas Universitarias y la CONEAU, se desarrolla un Sistema de Información para la Evaluación y el Mejoramiento Institucional (SIEMI). Este sistema, permite el registro y carga de información institucional prevista en la Resolución № 382/11, para el proceso de autoevaluación. Posteriormente la CONEAU aprobó un documento que ajusta y precisa los criterios y procedimientos para la evaluación externa por pares, la "Guía para la elaboración del informe del Comité de Pares Evaluadores".

De esta manera queda organizada la Evaluación institucional en dos etapas, la primera de autoevaluación que desarrolla la universidad (que incluye la carga 
del SIEMI y el documento de autoevaluación institucional) y la segunda de evaluación externa que desarrolla la CONEAU (que involucra un comité de pares evaluadores externos y la respectiva visita), para finalmente elaborar un Informe final de evaluación que incluye distintos juicios y recomendaciones, por parte del comité de pares evaluadores.

La primera etapa de la Evaluación Institucional es la Autoevaluación. El proceso de Autoevaluación Institucional es una instancia privilegiada de producción de conocimiento de y sobre la universidad, de carácter fuertemente colegiado y participativo de todos los integrantes de la comunidad universitaria. Dicho proceso requiere abordar, a partir de un relevamiento sistemático e integral de datos cuali y cuantitativos rigurosos, un análisis holístico e integrado de las dificultades y logros en el cumplimiento de las funciones sustantivas, teniendo en cuenta la mirada histórica, contextual y prospectiva. Es decir, dicho proceso debe permitir a la universidad la revisión analítica y objetiva de su proyecto y la proyección de su mejoramiento.

La segunda etapa de la Evaluación Institucional es la Evaluación Externa. Esta instancia ya no se encuentra en potestad de la institución universitaria, sino que es organizada por la CONEAU. A tal fin, según establecen las normas del procedimiento la CONEAU define y designa un comité de pares evaluadores externos, un equipo de técnicos y consultores para llevar a cabo este proceso. Dicho equipo es quien pautará la visita y las condiciones a desarrollarse en la misma, a partir del análisis del documento de autoevaluación y de los datos del SIEMI. La visita prevé en su agenda entrevistas con personal directivo de la institución, consejeros y otros actores integrantes de la comunidad universitaria (docentes, estudiantes, no docentes, egresados) así como con representantes de organizaciones profesionales, asociaciones civiles y autoridades 
gubernamentales de la región. De acuerdo a los procedimientos regulados, una vez efectivizada la visita se realiza el Informe preliminar, que es enviado a la institución para su opinión y consideración. A partir de la respuesta institucional a este informe preliminar la CONEAU elabora y aprueba con carácter de resolución el Informe Final en el plenario correspondiente.

\section{Acreditación carreras de grado}

\section{La normativa que regula los procesos de autoevaluación y acreditación de carreras de grado y la validez de títulos.}

Tal como se mencionó anteriormente el artículo 43 de la Ley de Educación Superior establece que los planes de estudio de carreras correspondientes a profesiones reguladas por el Estado, cuyo ejercicio pudiera comprometer el interés público, poniendo en riesgo de modo directo la salud, la seguridad y los bienes de los habitantes, deben tener en cuenta la carga horaria mínima, los contenidos curriculares básicos, los criterios sobre intensidad de la formación práctica, las actividades profesionales reservadas al título y los estándares establecidos por la normativa del Ministerio en acuerdo con el Consejo de Universidades.

La acreditación involucra dos momentos, el primero referido a completar un proceso de autoevaluación a partir de instrumentos específicos (CONEAU Global), el segundo una evaluación externa de pares que analiza el informe de autoevaluación respecto de los estándares definidos como parámetros de condiciones mínimas, de cumplimiento por parte de la carrera. Producto de lo cual, la CONEAU emite un informe de acreditación, y posteriormente el Ministerio la Validez Nacional del título. 
En los últimos años se produjo un cambio fundamental en la manera de definir los instrumentos para la acreditación de las carreras incluidas en el artículo 43. En primer lugar, mediante la redefinición de criterios para la formulación de actividades reservadas y su posterior revisión conjunta. En segundo término, mediante la redefinición de los criterios para la elaboración de estándares de acreditación.

El nuevo marco normativo entonces establece que, la acreditación de carreras de grado no es una medida relacionada con la evaluación del funcionamiento de las instituciones universitarias y de su calidad, sino, de manera específica, con la garantía de una formación idónea para el desempeño en actividades de riesgo público.

En el año 2019 la Resolución MECCYT N 1051 aprueba el "Documento de estándares de aplicación general para la acreditación de carreras de grado”, que constituirá la base para la elaboración de los estándares de acreditación específicos para cada carrera de grado ya incorporada o que se incorpore al régimen del artículo 43 de la LES.

Los estándares establecen los requisitos que se consideran mínimos e indispensables para una formación profesional que garantice el resguardo del interés público, respecto de las actividades de riesgo. Se agrupan en cinco dimensiones: 1) Condiciones Curriculares, 2) Condiciones para la Actividad Docente, 3) Condiciones para las Actividades de los Estudiantes, 4) Condiciones de Evaluación y 5) Condiciones Organizacionales. Estas condiciones se complementan con los requisitos establecidos en el artículo 43: carga horaria mínima, contenidos curriculares básicos e intensidad de la formación práctica. Cada uno de estos componentes se definen atendiendo a las actividades de riesgo y no a la totalidad de alcances de la titulación. 


\section{Pensar los procesos de acreditación de carreras de grado}

La propuesta que se presenta a continuación que involucra el diseño de un proyecto de intervención concreta en el desarrollo de los procesos de acreditación de las carreras de grado de la UNLP y atiende su integración con el proceso de Evaluación Institucional.

Se desarrolla en dos niveles:

A) Desarrollar un diseño metodológico de producción de información permanente de las distintas dimensiones para los procesos de acreditación de las carreras de grado.

B) Diseñar estrategias y elaborar materiales de capacitación para los procesos de acreditación de las carreras de grado.

A) Producción de la Información de las distintas dimensiones

Se detallan, agrupadas en Dimensiones, las condiciones que se consideran mínimas indispensables para la formación profesional que resguarde el interés público. Para el proceso de acreditación esas condiciones se complementan con las cargas horarias mínimas, los contenidos curriculares básicos y la formación práctica.

Cada una de las condiciones, incluyen varios componentes que se definen a continuación:

1. Las Condiciones Curriculares incluyen las especificaciones de los planes de estudios, los espacios curriculares, los programas incluyendo las cargas horarias, los contenidos mínimos y las características de la formación práctica. Identifica, además, las comisiones de planificación y seguimientos de los planes de estudios. 
2. Las Condiciones para la Actividad Docentes incluyen a todos los docentes de la carrera. Debe considerarse como criterio general que el cuerpo académico debe cubrir la totalidad de funciones sustantivas de la unidad académica. Es importante contar con equilibrio entre la formación académica, la dedicación y la participación de los docentes en actividades de investigación, extensión y docencia. Es necesario tener en cuenta: la cantidad de docentes, los cargos, la titulación, la categoría, la dedicación, el desarrollo de sus actividades en pregrado, grado o posgrado. Además, su desempeño en actividades docencia, de investigación, extensión desarrolladas en el ámbito de la institución o asociada a otras instituciones y la cantidad de docentes investigadores categorizados (programa de incentivos del Ministerio de Educación, CONICET u otros)

Se deberán tener en cuenta los mecanismos de selección, ingreso, permanencia y promoción de los docentes (concurso público de antecedentes y oposición para cargo ordinario, concurso de antecedentes y oposición para cargo interino, designación interina, contratación para actividad específica), las actividades de capacitación y evaluación docente tanto en las disciplinas específicas, como en lo relacionado con la enseñanza, la evaluación y el curriculum.

3. En las Condiciones para la Actividades de los Estudiantes los criterios para el ingreso y el número total de estudiantes deben ser coherentes con el proyecto académico y con la formación del perfil propuesto por las distintas carreras.

Se deberán analizar los requisitos generales y específicos para el ingreso, la información consolidada del número de aspirantes, ingresantes y alumnos de 
las carreras. Se deberán analizar las políticas de retención estudiantil (acciones de apoyo y orientación a la formación de los estudiantes, fortalecer su capacidad académica, planificar sus actividades o acceder a ámbitos y recursos que apoyen sus tareas) y las políticas de bienestar estudiantil (incluyendo becas).

Este criterio incluye la participación de estudiantes en actividades, proyectos y programas de investigación y extensión, como la existencia de acciones, políticas o programas propios o no destinados a promover dicha participación.

4. Las Condiciones de Evaluación identifican la existencia de procedimientos periódicos para revisar las actividades de evaluación de los aprendizajes de los estudiantes y de la comunicación de los resultados. Se debe tener en cuenta la existencia de actividades, mecanismos y/o instancias para la sistematización de información académica de los estudiantes, datos de deserción, desgranamiento y el tiempo empleado para la realización de la carrera.

Con respecto al seguimiento de sus graduados, este criterio debe identificar las acciones y/o instancias que utiliza la carrera (o como parte de una instancia mayor), para obtener, sistematizar y analizar información sobre sus graduados Se debe tener en cuenta también la participación de los alumnos y los graduados como elemento para evaluar los procesos de formación.

5. Las Condiciones Organizacionales incluyen la especificación de las instancias de gestión académica necesarias para el cumplimiento de las actividades de formación (estructura organizativa, estructura de gobierno) y los convenios y/o acuerdos interinstitucionales que tiene la carrera por si misma o por parte de una unidad mayor para el desarrollo 
de los proyectos vinculados con la actividad de docencia, investigación y extensión.

También incluye el acceso a sistemas de información para la gestión académica y administrativa que tiene la carrera.

Se deberán registrar el conjunto de edificios a través de propiedad, administración, usufructo, tenencia o por convenios interinstitucionales de la institución universitaria en el que se realizan tareas de gestión, administrativas, actividades académicas, recreativas, culturales y de servicios a la comunidad educativa, teniendo en cuenta su cantidad, capacidad y disponibilidad horaria para llevar adelante las distintas actividades que se desarrollan. Estos espacios y el equipamiento disponible deberán considerar el correcto desarrollo de las actividades de docencia, investigación y extensión. Se deberá tener en cuenta el acceso al acervo bibliográfico, a las redes de información y a los sistemas interbibliotecarios así como la modalidad de los préstamos, el horario de atención al público y a la comunidad académica, los usuarios, los servicios y los programas de capacitación que garantizan el uso adecuado para la implementación del proyecto académico

Además, es necesario contar con información de la unidad académica en que se inscribe de manera específica la carrera, para el desarrollo de su autoevaluación y articulada a la Evaluación Institucional, referida entre otros temas a: Mesas de Trabajo, Actividades de Innovación, Consejo Social, Consejos Consultivos, Relaciones Institucionales, Salud, Arte y Cultura, no incluidas en los estándares pero necesaria para el proceso de evaluación permanente de la institución atendiendo a los lineamientos del Plan Estratégico. 
B) Estrategias y materiales de capacitación para la acreditación de carreras de grado de la UNLP

A partir de la definición de las dimensiones, sus indicadores y algunos criterios, en función del enfoque asumido, se proponen estrategias y materiales de capacitación para consolidar la conformación de equipos de evaluación para atender los procesos de acreditación.

Estos equipos deberían ser interareales e interdisciplinarios de cada unidad académica, contando con el asesoramiento de las áreas centrales específicas como el equipo profesional técnico de la DEySA y los responsables de las distintas áreas de las Unidades Académicas encargadas de dar respuesta a las distintas dimensiones.

Las etapas serían:

1) Creación de áreas de acreditación y evaluación o designación de responsables de dichos procesos en cada una de las unidades académicas, para ir consolidando equipos de especialistas en el tema.

2) La definición de una agenda de trabajo.

3) El diseño de un sistema de información para la acreditación, con interfaces entre los sistemas de información hoy disponibles y en concordancia con el sistema de información para la Evaluación Institucional permanente de la UNLP.

4) Actualización, revisión o corrección de los datos hoy disponibles.

Para la implementación de estas estrategias y el desarrollo de los procesos de acreditación se requerirá contar con materiales de capacitación específicos. Será necesaria la definición de un programa de cursos y capacitaciones presenciales y online que permitan acercar posibilidades de formación 0 
actualización en la temática a los integrantes de los equipos técnicos de las unidades académicas.

Serían objetivos estratégicos:

1) Capacitar a los equipos de las unidades académicas o dependencias en temas de acreditación.

2) Generar materiales y cursos de capacitación en temas específicos, relacionados a los procesos acreditación de la CONEAU.

3) Publicar y dar a difusión materiales y desarrollos de la UNLP.

\section{Reflexiones finales}

Todo proceso evaluativo involucra una producción de conocimiento sobre el que se emitirán juicios de valor respecto de un criterio o parámetro (externo, propio o histórico). Por otra parte, la comprensión de que la evaluación representa una elaboración conceptual cuyo objeto de conocimiento es la práctica inmediata, sea esta en el nivel del aprendizaje o en el nivel institucional. Sin embargo, la producción de conocimiento sobre sí que tiene la universidad, en general se ha ido desarrollando, atendiendo a diversas demandas internas y externas, a las cuales no rige el mismo sentido o construcción de criterios.

Es por ello por lo que se considera central generar políticas integrales de evaluación que permitan que la misma se consolide como un insumo permanente de la gestión estratégica ya sea esta en el nivel institucional o en el del desarrollo específico de carreras, ya que es la forma privilegiada que tiene la institución para, desde la gestión y el planeamiento, generar transparencia y 
rendir cuenta sobre sus funciones sustantivas, entendiendo que la educación es un bien público y social y un derecho humano universal.

\section{Bibliografía}

- Catino, Magali (2020) La Planificación estratégica y la gestión de la Evaluación Institucional como política permanente de mejoramiento de la Universidad Nacional de La Plata para el período 2018-2022. http://sedici.unlp.edu.ar/handle/10915/115503

- Estatuto de la Universidad Nacional de La Plata, 2008.

- Normativa CONEAU, https://www.coneau.gob.ar/?page id=257

- Plan Estratégico de la UNLP 2018 - 2022

- Semper, Marina (2020) La planificación estratégica de los procesos de acreditación de las carreras de grado de la UNLP como política permanente de mejoramiento http://sedici.unlp.edu.ar/handle/10915/115641

- Tauber, F. (2008). I. La planificación, la gestión, la comunicación institucional. Consideraciones en torno al estado de la cuestión referido al objeto de estudio y su contexto. En La comunicación en la planificación y gestión para el desarrollo de las instituciones universitarias públicas argentinas: el caso de la Universidad Nacional de La Plata en el trienio junio 2004-mayo 2007 (Tesis de Doctorado). Facultad de Periodismo y Comunicación Social - Universidad Nacional de La Plata, La Plata.

- Tauber, F. (2018). Pensar la Universidad. Proyecto institucional de la Universidad Nacional de La Plata 2018-2022 